\title{
Archéopages
}

Archéopages

Archéologie et société

46 | 2018

Maisons

\section{Un quartier de la ville antique de Vesontio (Besançon)}

L'imbrication de l'habitat et des activités commerciales

A district of the ancient city of Vesontio (Besançon). The intertwining of housing and commercial activities

Un barrio de la ciudad antigua de Vesontio (Besanzón). La imbricación del

hábitat y de las actividades comerciales

Christophe Gaston et Claudine Munier

\section{OpenEdition}

Journals

Édition électronique

URL : https://journals.openedition.org/archeopages/3950

DOI : 10.4000/archeopages.3950

ISSN : 2269-9872

Éditeur

INRAP - Institut national de recherches archéologiques préventives

Édition imprimée

Date de publication : 1 décembre 2018

Pagination : $36-43$

ISSN : 1622-8545

Référence électronique

Christophe Gaston et Claudine Munier, "Un quartier de la ville antique de Vesontio (Besançon)», Archéopages [En ligne], 46 | 2018, mis en ligne le 01 décembre 2020, consulté le 02 juin 2021. URL : http://journals.openedition.org/archeopages/3950 ; DOI : https://doi.org/10.4000/archeopages.3950 


\title{
Un quartier de la ville antique \\ de Vesontio (Besançon) \\ L'imbrication de l'habitat \\ et des activités commerciales
}

\author{
Christophe Gaston Inrap, UмR 6249 «Chrono-environnement" \\ Claudine Munier Ville de Besangson, UMR 6249 «Chrono-environnement "
}

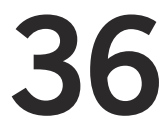

1. Fouille réalisée par le service municipal

d'archéologie de la ville de Besançon en collaboration avec

l'Inrap, sous la

responsabilité de

Claudine Munier.

La fouille a été complétée par une étude du bât

au printemps 2011

(Viscusi-Simonin, 2016).
Une fouille archéologique a été menée sur un périmètre de près de 4 ooo $\mathrm{m}^{2}$ entre 2010 et 2012 dans la Zac Pasteur à Besançon' (Munier, 2016), dans la partie basse de la ville antique, en rive gauche du Doubs, non loin du cardo et à proximité de l'ancien pont romain, alors unique franchissement en pierre de la rivière [ill. 1]. L'étude de l'occupation gallo-romaine de ce quartier renouvelle nos connaissances sur l'urbanisation de Vesontio, dans le sens où sa création et son développement ne peuvent être comparés à ceux d'autres quartiers antiques déjà étudiés dans la ville.

\section{Création d'un nouveau quartier à l'Antiquité}

L'originalité réside essentiellement dans le fait qu'il s'agit d'un quartier créé vers 40 de notre ère, en une seule phase, sans doute à la suite d'une volonté politique précise : occuper ce secteur stratégique situé en tête de pont et y développer une activité résidentielle et commerçante. Si de rares structures d'habitat plus anciennes préexistent, elles ne sont pas antérieures au début de notre ère et ne pérennisent pas, comme ailleurs dans la ville, des occupations laténiennes (D1 et D2 essentiellement) de l'oppidum. Le caractère très humide du secteur (présence de paléochenaux et proximité de la rivière) explique l'absence d'occupations gauloises. L'apport de remblais importants pour isoler ce nouveau quartier de ces désagréments, ainsi que l'homogénéité des maçonneries des différentes maisons galloromaines et de l'organisation parcellaire confirment la cohérence de cette nouvelle urbanisation. Si les vestiges découverts dans certains autres secteurs de Vesontio ont engendré un stéréotype maintes fois propagé par les acteurs de la recherche, celui d'un arrêt du développement urbain à la fin du $\mathrm{II}^{\mathrm{e}}$ siècle à la suite d'une urbanisation intense aux $\mathrm{I}^{\mathrm{er}}$ et $\mathrm{II}^{\mathrm{e}}$ siècles, les maisons fouillées à la Zac Pasteur donnent une tout autre image : celle d'un dynamisme urbain qui ne faiblit pas entre 40 et la fin du $\mathrm{III}^{\mathrm{e}}$ siècle, voire le milieu du IV ${ }^{\mathrm{e}}$ siècle pour les parcelles situées au plus près du cardo. On ne peut pas alors faire l'impasse du lien possible, bien qu'il reste à être démontré, entre le « castrum » de l'Antiquité tardive et le quartier du bourg médiéval en tête de pont. Mais revenons à nos maisons...

\section{Propriété et statut social}

Douze parcelles ont pu être définies dans l'emprise du chantier. L'évolution de chacune d'elles illustre le développement d'un quartier qui peut sembler modeste à l'origine, mais qui se densifie progressivement [ill. 2]. La propriété 11, avec sa domus à péristyle de plus en plus luxueuse, en est l'image la plus parlante, même si elle n'est pas comparable aux autres propriétés. Une limite physique se dessine ainsi au sein du parcellaire, entre deux catégories sociales : les parcelles tournées vers la rue (2 à 10) sont occupées par des maisons de commerçants et d'artisans, alors que celles tournées vers le Doubs (11 et 1) appartiennent à des domus plus vastes, demeures de familles plus aisées.

Chaque parcelle est limitée par un mur mitoyen, dont l'omniprésence serait peut-être un indice de la précocité du parcellaire dans le secteur, comme cela est supposé pour les agglomérations de Belgique (Brulet, 2008, p. 97). La puissance des fondations de ces murs suggère qu'ils constituaient un véritable paries communis, c'est-à-dire un murporteur construit d'un commun accord entre deux propriétaires ou hérité d'un choix antérieur (Berti 

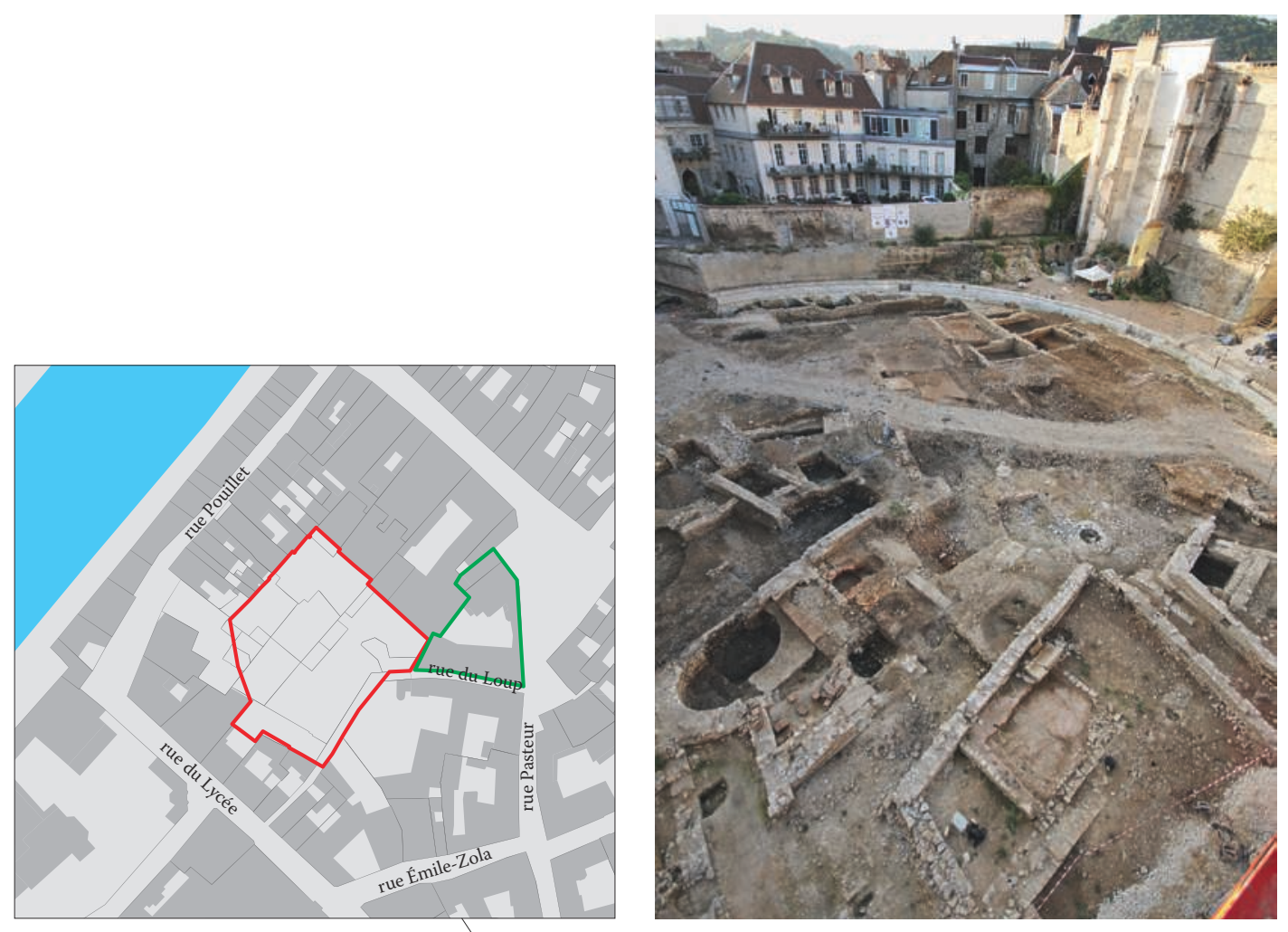

1. Localisation de la Zac
Pasteur et vue des structu

gallo-romaines depuis l'ouest.

Outre les maisons gallo-

romaines, la stratigraphie

(6,50 m de hauteur) a livré

un cimetière daté entre

la fin du vII' et le XII siècle,

des structures artisanales

ou agricoles à l'arrière de

I'habitat de la fin du Moyen

Âge, et enfin les jardins

d'hôtels particuliers

à l'époque moderne.

— fouille

— étude du bâti

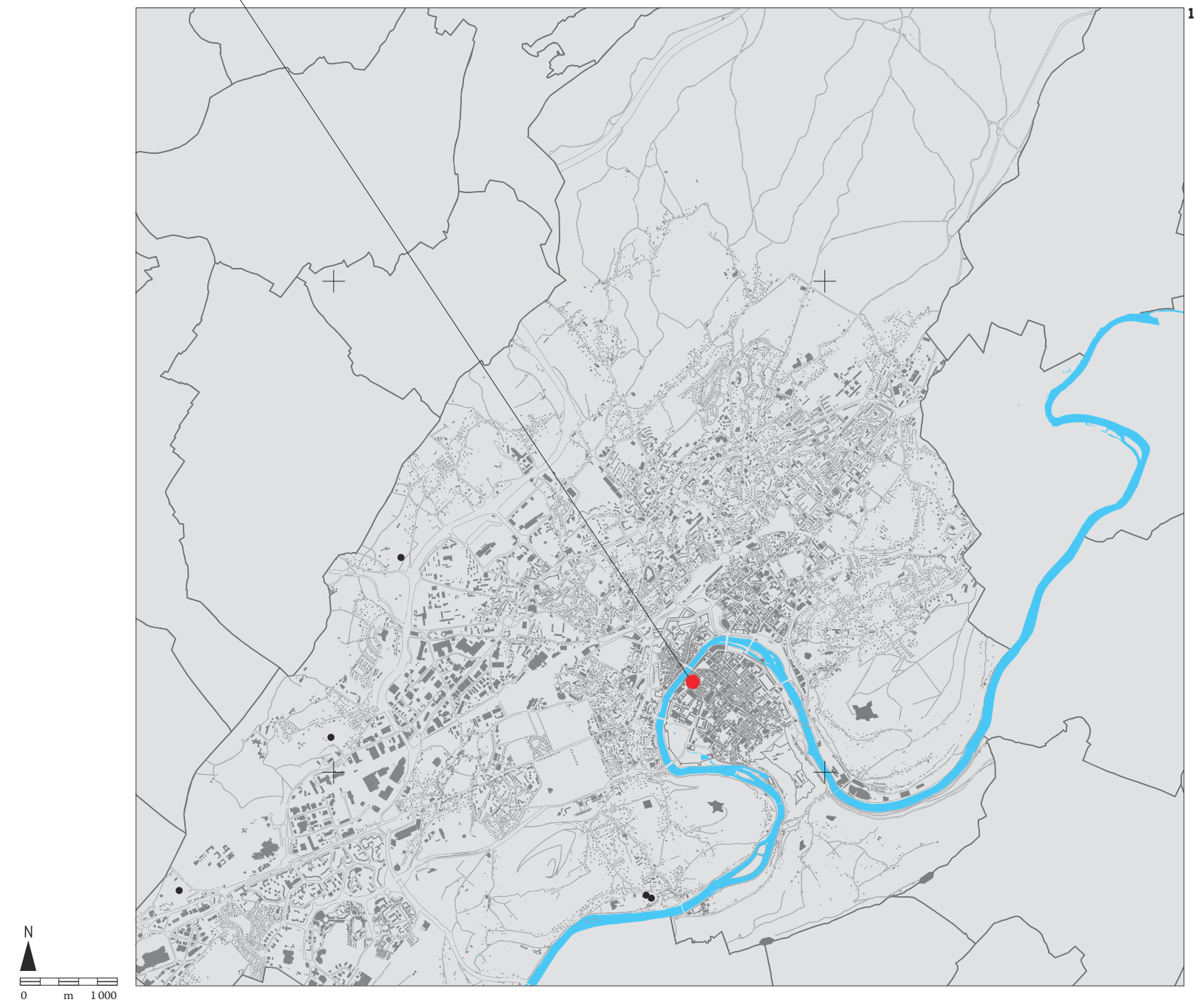




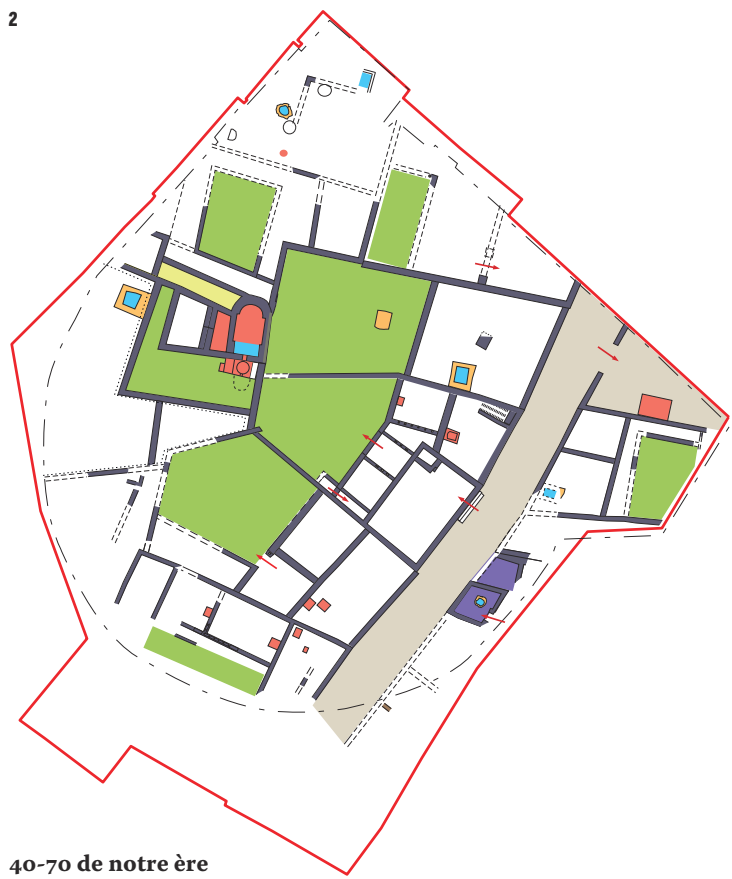

$\stackrel{\infty}{\infty}$

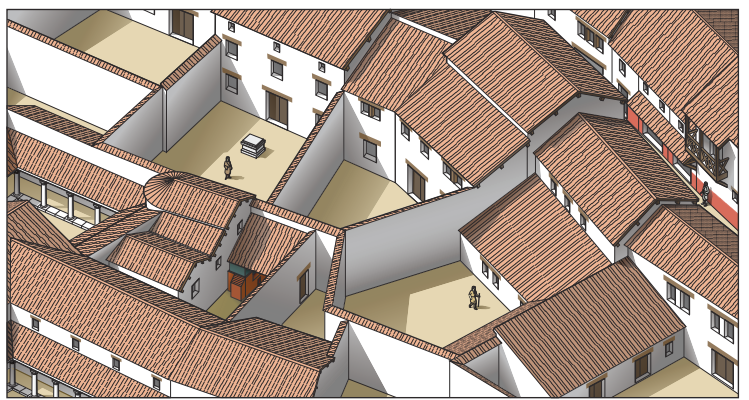

Rossi, May Castella, 2005, p. 258 ; Brulet, 1996, p. 94). La particularité remarquable de ces murs, du moins dans la première phase d'urbanisation (vers 40), est que les frais de la construction des murs mitoyens sont toujours partagés par les propriétaires des deux parcelles contiguës : ainsi, un propriétaire construira par exemple le mur mitoyen nord et laissera la construction du mur mitoyen sud-est à la charge de la parcelle voisine au sud, comme on le constate sur certaines parcelles d'Herculanum (Monteix, 2010, p. 362). C'est ici un témoignage concret de la notion de servitus tigni immitendi (Saliou, 1994, p. 39). On retrouve un exemple identique de droit du voisinage à Bliesbruck, où la limite parcellaire tournée vers le cardo est à la charge du voisin (Petit, Santoro, 2007, p. 93).

La largeur des façades varie ici du simple au double (8,5 à 20 m), s'inscrivant dans les largeurs moyennes (6 à $20 \mathrm{~m}$ ) en façade des parcelles relevées en Gaule du Nord-Est (Coquelet, 2011, p. 94). Quant à la profondeur maximale de ces mêmes parcelles, elle est comprise entre 22 et $30 \mathrm{~m}$, valeur se situant plutôt dans la moyenne basse des dimensions observées ailleurs : en Gaule du Nord-Est, elle est généralement comprise entre 30 et $40 \mathrm{~m}$ (Coquelet, 2011, p. 97). Les parcelles
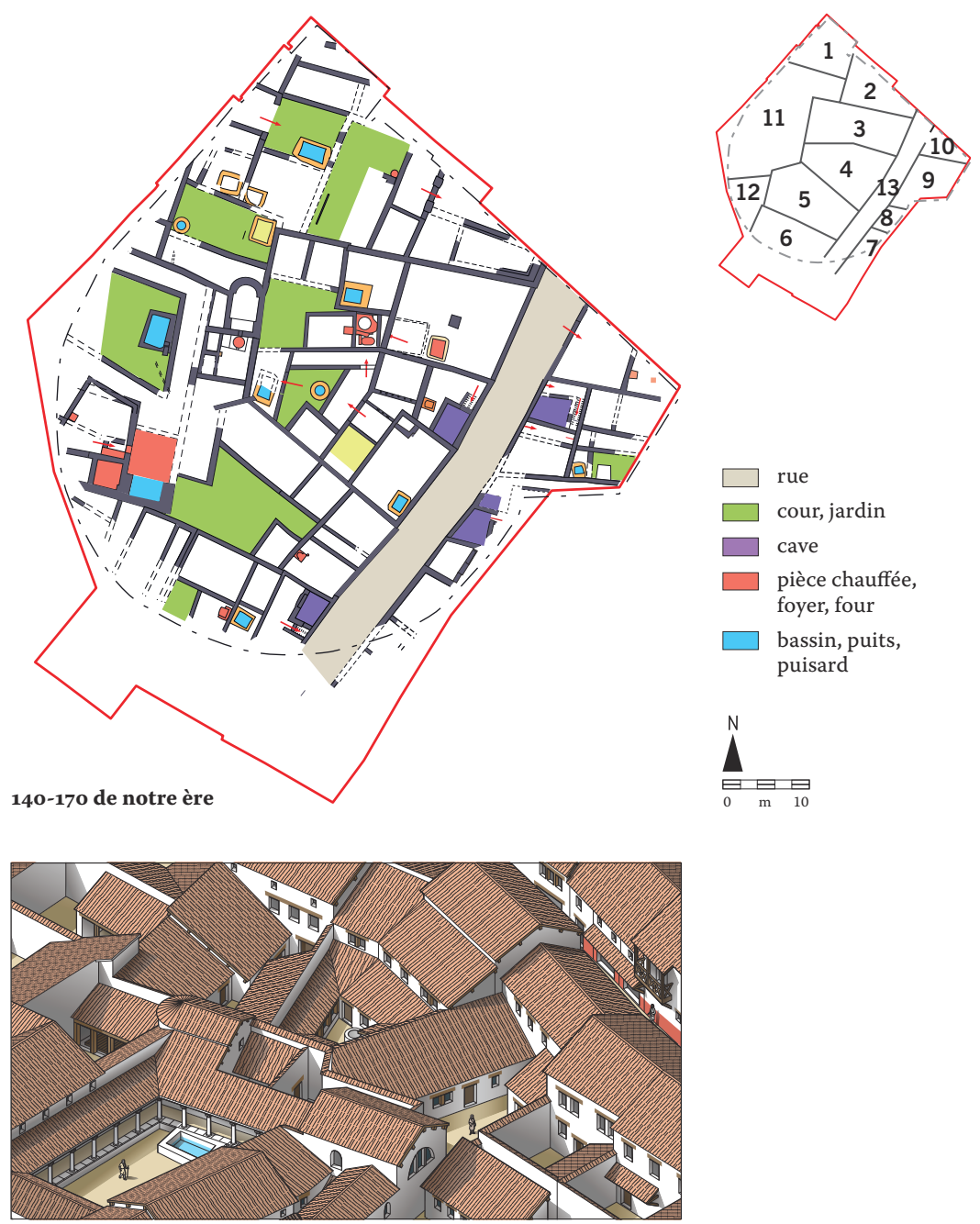

atteignent à Besançon 300 à $400 \mathrm{~m}^{2}$, la moitié au moins de la surface de chacune d'elles étant occupée par la maison. Nous aurions donc ici un lotissement offrant des maisons de plan allongé mitoyennes présentant leur petit côté à la rue, dans lequel cependant des propriétés plus larges seraient occupées par de plus grandes domus, comme on l'observe pour la parcelle 11. Cependant, l'évolution parcellaire montre que la frontière fixée par la parcelle initiale est parfois franchie, et l'extension de l'espace nécessaire se fait alors au détriment de la propriété voisine, comme pour la domus de la parcelle 11, selon un usage assez habituel (Brulet, 1996, p. 95), ou, tardivement, par le regroupement de deux parcelles voisines (9 et 10 par exemple).

\section{Espace de vie, espace de travail}

Au sein de chaque parcelle, l'organisation de l'espace obéit à des règles assez fluctuantes. On trouve toujours les bâtiments principaux en bordure de rue. Dans six de ces bâtiments, une cave maçonnée est installée, toujours construite le long de leur façade sur rue. C'est sur l'espace libre laissé à l'arrière de la parcelle que vont se développer et s'étendre les annexes et rajouts successifs, jusqu'à occuper presque entièrement
2. Exemple de densification de l'habitat: relevé des structures entre $\mathbf{4 0}$ et 70 de notre ère et entre 140 et 170 et essais de restitution du quartier (vue depuis l'ouest), qui montrent l'agrandissement de la partie couverte par rapport aux espaces ouverts à l'arrière. 
l'espace disponible, parfois non sans difficulté. On retrouve ce principe dans nombre de villes antiques (Brulet, 2008, p. 100 ; Berti Rossi, May Castella, 2005, p. 212 ; Binet, 2010, p. 305-307).

Les cours à l'arrière des parcelles semblent toujours liées, pour les maisons donnant sur la rue, à des fonctions utilitaires, alors que, dans le cadre de la domus 11, cette zone, considérée comme un espace de détente et de représentation, est occupée par un jardin d'agrément (viridarium avec bassin) avec péristyle (ambulatio), dans le fond duquel un balneum privé est construit, renforçant ainsi cette volonté de tendre vers l'otium.

La hauteur minimale des bâtiments sur rue est déterminée par celle des constructions successives qui viennent s'accumuler sur leur façade arrière. En fonction des données, cette hauteur peut être estimée à environ $8 \mathrm{~m}$ en moyenne pour les murs gouttereaux, ce qui n'a rien d'exagéré (Olivier, 1988, p. 74-78; Kerébel, 2001, p. 129). Les toitures sont orientées logiquement avec les gouttereaux en façade (Olivier, 1988, p. 74-78), solution permettant non seulement d'évacuer plus facilement les eaux pluviales, mais également indispensable dans une région soumise à des précipitations neigeuses (Petit, Santoro, 2007, p. 110). La taille de ces bâtiments génère par conséquent une hauteur importante des murs de clôture à l'arrière des parcelles, ce qui reste tout à fait plausible, même en contexte rural (Provost, 2007, p. 88).

La distribution des fonctions n'est pas cloisonnée : on constate une imbrication des espaces d'habitat et de locaux artisanaux, qui rend souvent difficile la distinction des deux fonctions (Berti Rossi, May Castella, 2005, p. 213). Cependant, la deuxième a tendance à être davantage mise en avant par rapport à la première, comme on le constate ailleurs (ibid., p. 221 ; Coquelet, 2011, p. 187). On peut même parfois observer une subdivision supplémentaire en deux pièces au sein des locaux artisanaux, l'espace en contact direct avec la rue étant considéré alors comme un lieu de vente, et l'autre étant consacré à la production et au stockage (ibid., p. 187). Nous aurions peut-être ici une variation de ce même système, puisque les maisons sur rue, à l'exception d'une parcelle, présentent deux pièces en façade.

Aucun seuil n'étant conservé, on ne peut que se perdre en conjectures concernant les ouvertures liées aux usages et aux accès de ces bâtiments, d'autant qu'aucune galerie n'est attestée sur la rue. Ces ouvertures devaient se développer selon une gamme allant de la large baie de boutique jusqu'à la simple porte d'accès aux pièces du bâtiment (Monteix, 2010, p. 59). Quelques hypothèses peuvent être émises. Ainsi, la parcelle 5 dans la seconde moitié du $\mathrm{II}^{\mathrm{e}}$ siècle [ill. 2] offre une disposition qui ne semble pas poser trop de problèmes au regard d'autres plans de bâtiments déjà observés par ailleurs : un couloir central, donnant accès aux pièces à l'arrière de l'habitation, encadré par deux pièces de façade pouvant être interprétées comme des boutiques ou ateliers. On peut raisonnablement penser que le rez-dechaussée de cette façade devait être composé d'une porte axiale s'ouvrant sur le couloir, entre deux larges baies ouvrant sur les boutiques. La parcelle 4 est plus complexe [ill. 2] : une large ouverture se devine, soulignée par la présence d'un muret installé en saillie sur la chaussée de la rue. Cette dernière caractéristique (évoquant un large massif d'escalier), ainsi que l'aspect particulièrement soigné de la grande pièce à laquelle cette ouverture donne accès, laissent penser que cette baie devait présenter une qualité architecturale indéniable. L'interprétation de la partie nord-est de cette parcelle est moins évidente. La position d'un massif large d'1,30 m, appuyé sur l'angle interne du bâtiment, et doublant la largeur du mur de façade, suggère la présence d'un départ d'escalier desservant l'étage et accessible directement depuis la rue. Cet escalier évoque des aménagements semblables mis en évidence à Saint-Romain-enGal (Brissaud, 1999, p. 51), Pompéi (Adam, 1989, p. 340-350) ou Herculanum (De Vos, 1982, p. 254). Si ce massif correspond bien à ce type d'installation, il constitue un exemple intéressant d'un lotissement de la propriété urbaine aussi bien en plan qu'en élévation, avec sans doute la création d'au moins un appartement indépendant à l'étage (Monteix, 2010, p. 84). Dans la parcelle 8, l'organisation de l'espace situé au-dessus de la cave sud est sans doute liée au commerce : en effet, l'axe du mur de fond de la cave est repris par le cloisonnement partiel du foyer lors des phases ultérieures, suggérant un espace sur plancher (boutique) et une arrière-boutique (foyer, muret). Ce même principe se rencontre également dans la parcelle 6 : une cloison construite sur le mur de fond de cave indique qu'un petit espace était préservé le long de la rue (boutique?), alors que l'espace à l'arrière s'agrandissait avec un foyer central (atelier ?) [ill. 2 et 3]. On retrouve ici, du moins dans cette phase, une configuration évoquant celle des maisons de Bliesbruck (Moselle), à savoir un espace étroit en façade, occupé par une ou deux boutiques, et un espace plus vaste en retrait, donnant accès à des petits locaux (habitation, ateliers) à l'arrière (Petit, Santoro, 2007, p. 123).

\section{Quelles activités pour quelles parcelles?}

Les deux principales activités rencontrées dans cette partie du quartier, d'une part les métiers de bouche (commerce), d'autre part le travail du métal (artisanat), se côtoient et se croisent régulièrement au sein d'une même parcelle, dans les pièces en bord de rue ou dans les annexes situées à l'arrière [ill. 3]. Les caves construites en bord de rue sont généralement attribuées aux maisons abritant des boutiques ou des ateliers.

Les métiers de bouche regroupent le stockage alimentaire, la boulangerie, la meunerie, la fumaison-salaison et la restauration (auberge). 
3. Parcelles en bord de

rue vers $\mathbf{2 0 0}$ de notre ère :

fumoirs, hypocauste,

auberge avec cheminée et

sols en béton de terrazzo

(en orange). Quelques-unes

des fosses maçonnées

quadrangulaires peu

profondes sont interprétées

comme des celliers, pour

lesquels I'usage domestique

ou commerçant ne peut

être précisé.

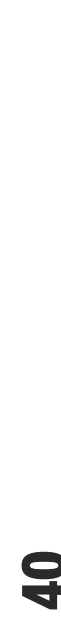

우
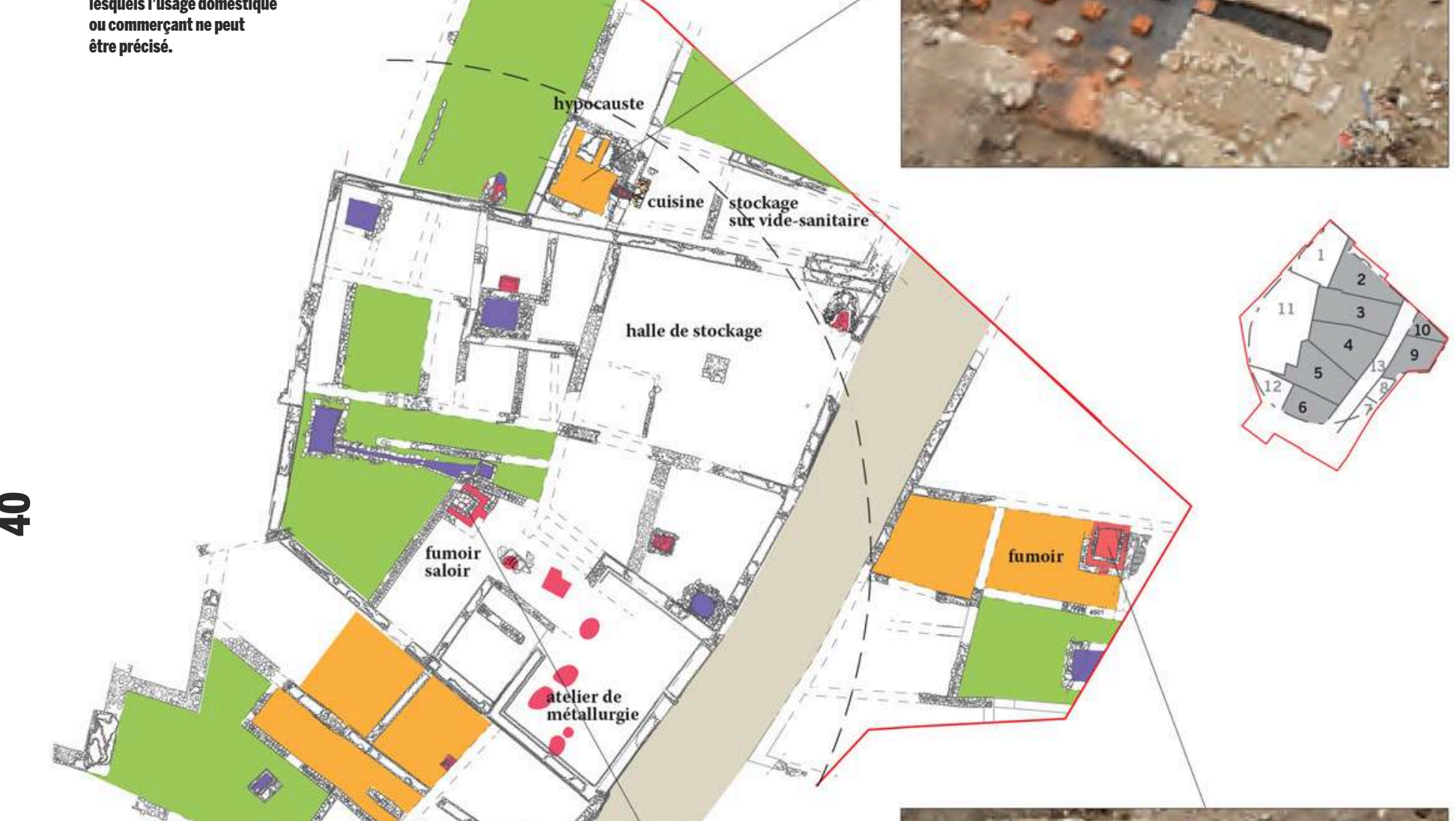

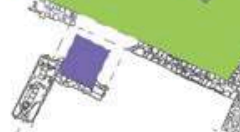
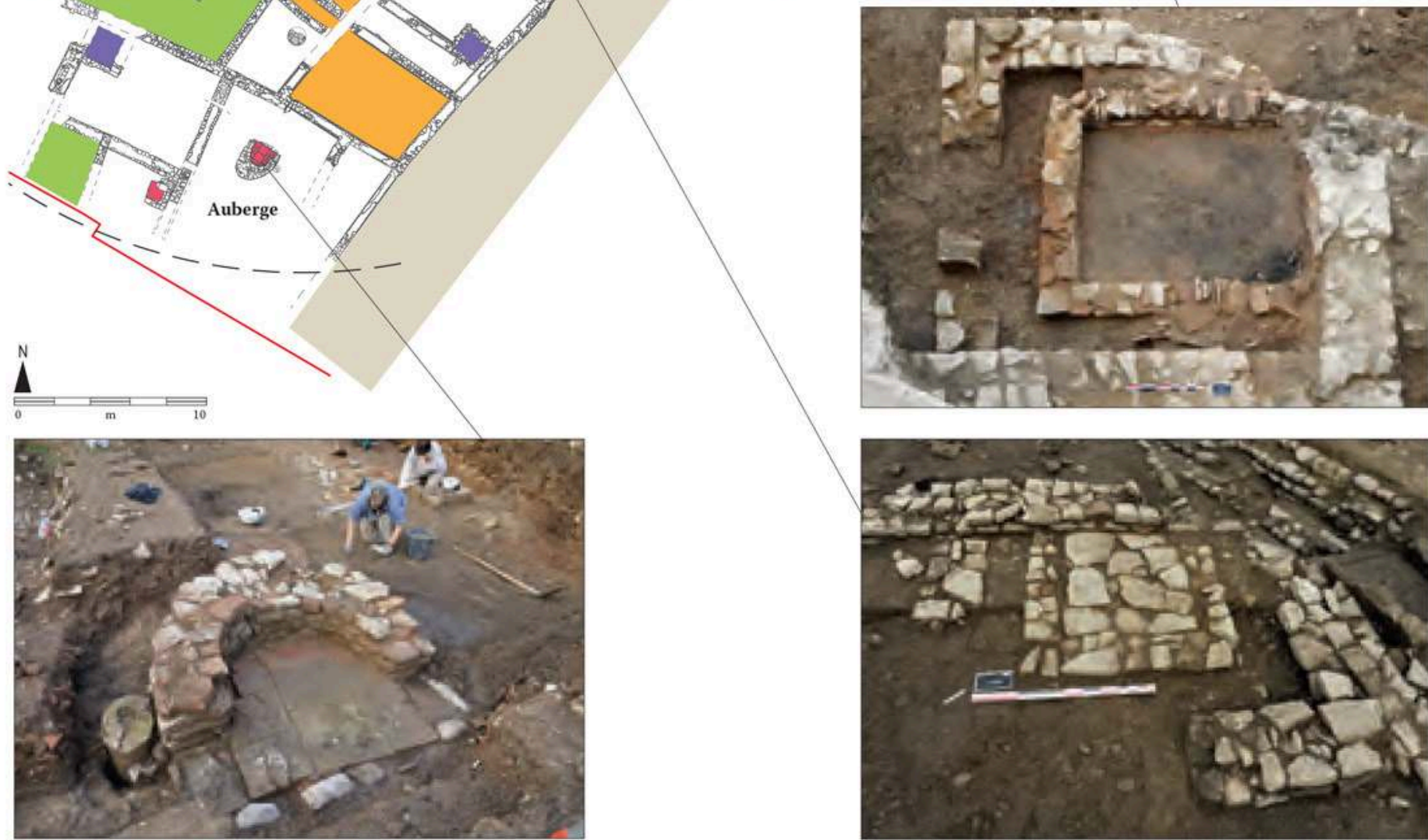


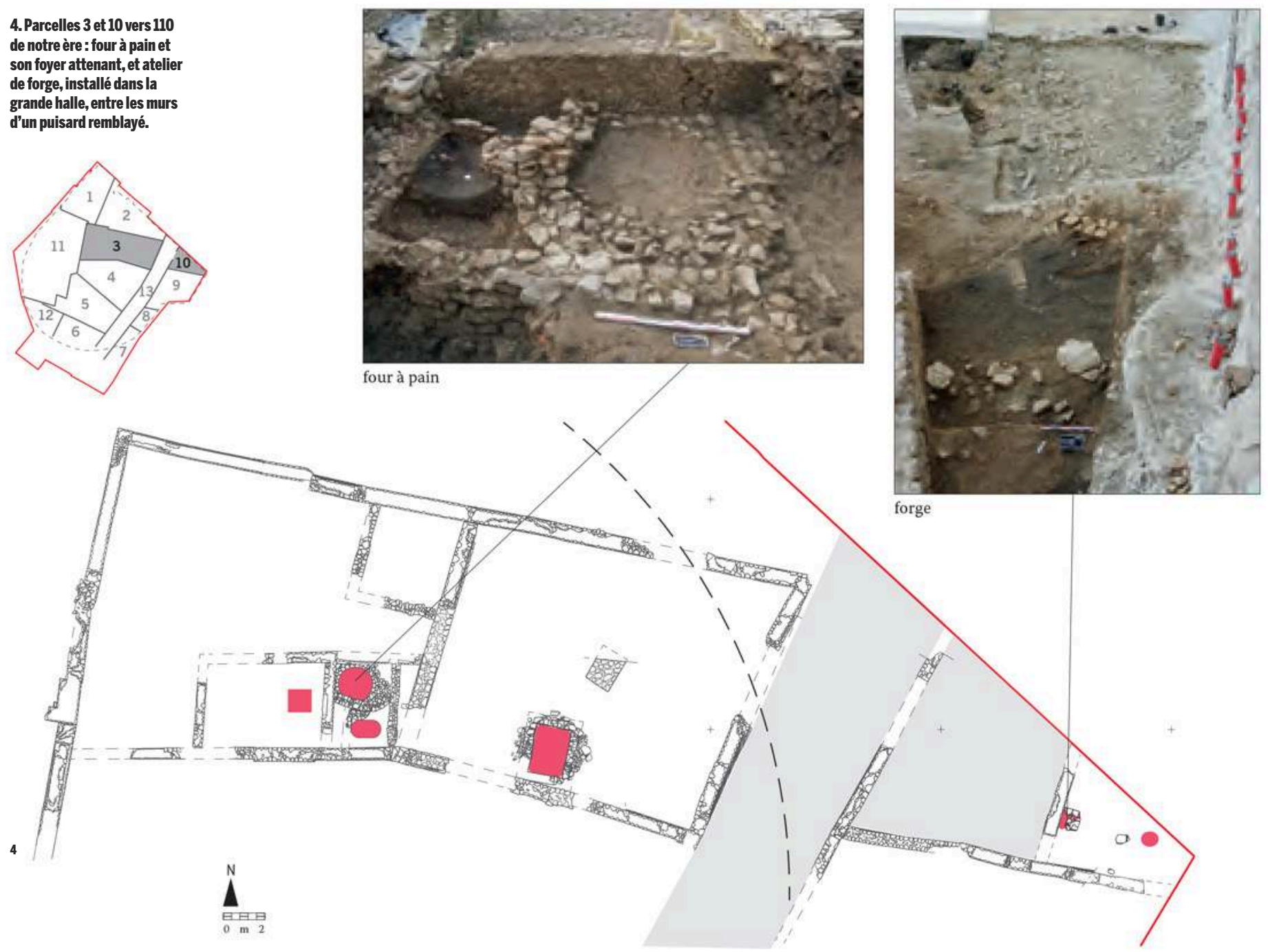

D'une trentaine de mètres carrés, la pièce à plancher sur vide sanitaire de la parcelle 2, destinée à stocker et conserver des réserves alimentaires, pouvait accueillir des produits très divers, évoluant selon les saisons. Construit vers 150, cet espace est chauffé par les foyers successifs d'une pièce arrière : d'abord celui d'un petit atelier de métallurgie, puis vers 200 celui d'une table de cuisson (cuisine) associée au praefurnium d'un hypocauste, enfin vers 250 par celui d'un nouvel atelier de travail du métal. La grande halle de la parcelle $3\left(140 \mathrm{~m}^{2}\right)$, édifiée dès 40 , n'est pas subdivisée avant le milieu du $\mathrm{III}^{\mathrm{e}}$ siècle. Entre 110 et 170, elle est ouverte sur une petite pièce arrière qui abrite un four à pain de $2 \mathrm{~m}$ de diamètre intérieur [ill. 4], fonctionnant en même temps qu'un foyer de métallurgie. Plusieurs tables de cuisson dépendent soit de l'espace privé de la cuisine (parcelle 11, début $\mathrm{II}^{\mathrm{e}}$ siècle), soit de l'eśpace lié au commerce (parcelle 2, début $\mathrm{III}^{\mathrm{e}}$ siècle ou parcelle 3 au milieu de ce siècle, à l'emplacement du four à pain).

La meunerie est uniquement représentée par des fragments de meta et de catillus de meules manuelles, hydrauliques ou à traction animale, présentes en majorité dans les contextes du II ${ }^{\mathrm{e}}$ siècle, parfois recyclées dans les caves où elles peuvent être utilisées comme support. Si les meules manuelles servent dans l'espace domestique, les plus grandes sont en revanche destinées à une production plus importante, attestant un potentiel commerce de farine, dès l'époque flavienne en parcelle 8 , puis au $\mathrm{II}^{\mathrm{e}}$ siècle en parcelles 8,4 et $6^{2}$.

Installés dans les parcelles 4 et 9 au début du $\mathrm{III}^{\mathrm{e}}$ siècle, deux fumoirs constituent les premières installations de ce type trouvées à Besançon [ill. 3]. Loriginalité de celui de la parcelle 4 est de côtoyer directement une petite cuve reliée à un puisard par un caniveau maçonné, cuve qui pourrait correspondre à un saloir, attestant l'association salaison-fumaison. Quelques arêtes de poisson (indéterminé) ont été trouvées au fond de cette cuve. Aucun indice de boucherie n'a pu être mis en évidence.

Au moins deux cheminées monumentales (foyers ouverts en hémicycle) ont été fouillées (parcelles 8 et 6). Celle de la parcelle 6, la mieux conservée, possède deux états de construction situés entre 170 et 250 . Ses dimensions $(1,80 \mathrm{x}$ $2,20 \mathrm{~m}$ hors tout), sa sole en tuiles puis en briques dans le second état (réutilisation de bessales, pedales, sesquipedales) et son
2. Étude Luc Jaccottey, Inrap. 


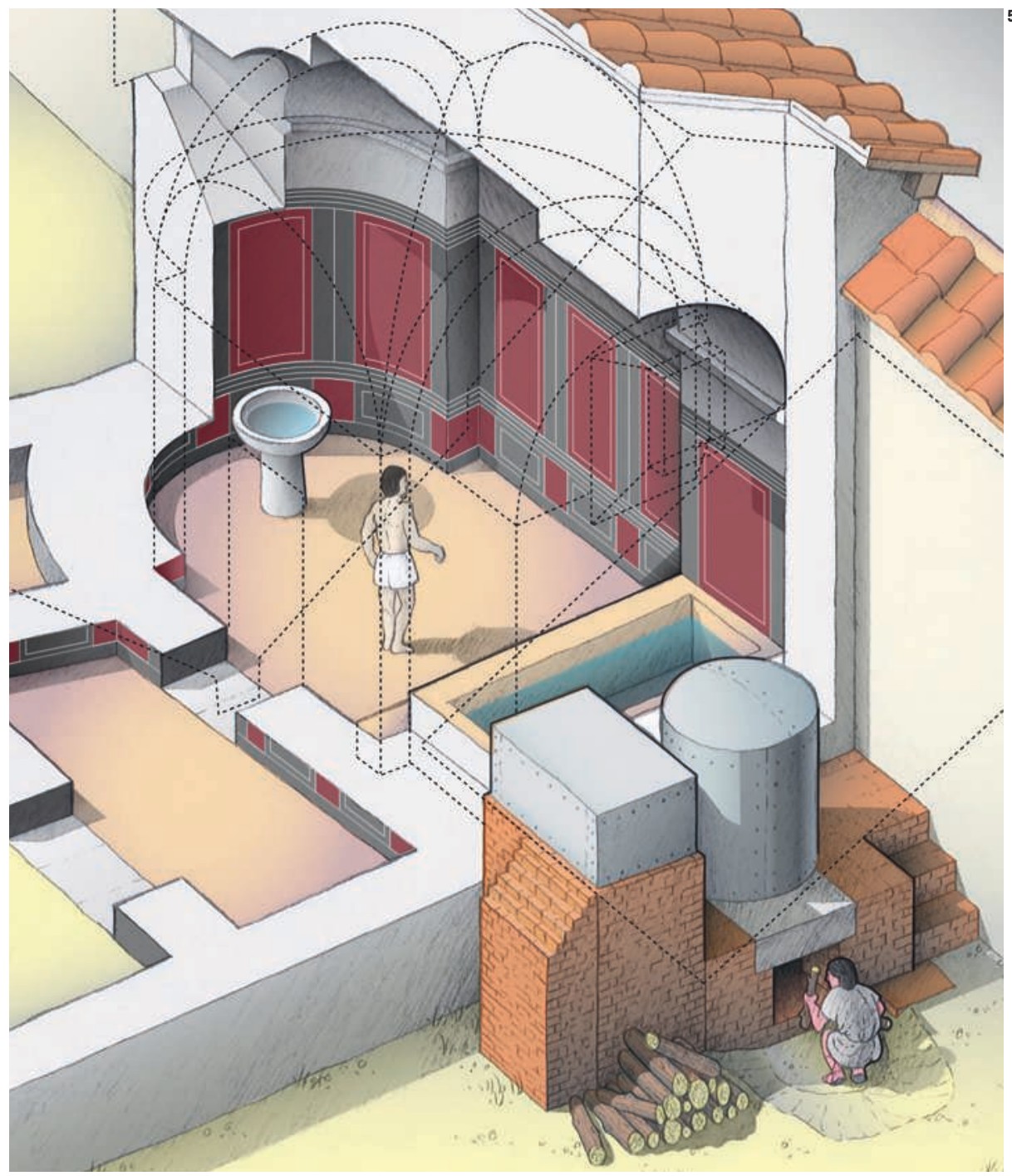

5. Essai de restitution du caldariumet de l'espace technique du petit balnéaire de la domus résidentielle 11 [voir ill.2].

contrecœur maçonné en font un aménagement particulièrement impressionnant [ill. 3]. Constitué d'un fragment de colonne recyclé, un support de potence avec crapaudine creusée en son centre borde la cheminée au nord. Les dimensions de la cheminée et la présence du support de potence suggèrent une utilisation destinée à une collectivité (auberge, taverne).

En complément de ces structures, le mobilier n'a pas permis, hormis les déchets de métallurgie, de préciser d'autres activités artisanales ou commerciales : de rares pesons sont bien insuffisants pour déceler autre chose qu'une activité domestique, tout comme on ne peut faire la part entre les objets (céramique, verre, métal ou os...) destinés à être vendus et ceux utilisés dans les habitations. La céramique découverte en rejet dans les fosses maçonnées abandonnées (puisards, celliers...), en quantité beaucoup plus importante dans les maisons de bord de rue que dans les domus résidentielles, pourrait cependant participer de cette dynamique commerciale.

À côté de ces métiers de bouche, l'activité

3. Étude CNRS. la plus courante, tout au moins celle qui a laissé le plus de traces, est le travail du métal (forge et travail des alliages base-cuivre) ${ }^{3}$. Toutes les parcelles, même la domus résidentielle de la parcelle 11, connaissent, au moins une fois dans leur évolution, la présence de cet artisanat suggérée par des résidus isolés (battitures, scories) ou par l'assemblage de ces déchets avec des fours ou des foyers attestant l'existence d'ateliers. Il semble que les ateliers de fabrication d'objets à vocation généraliste côtoient des ateliers plus spécialisés. Si, le plus souvent, cette activité semble destinée à alimenter des besoins ponctuels, quelques cas montrent, à travers l'extension des zones artisanales et leurs réaménagements successifs, des productions plus pérennes. Dans le cas de la parcelle 1o par exemple [ill. 4], c'est toute une pièce qui témoigne de cette activité, entre 70 et 170, à travers ses microcouches d'occupation, ses foyers, la répartition dans toute la pièce des battitures et la présence d'enclumes en pierre. Dans la parcelle 1 , cet artisanat est régulièrement déplacé entre différentes pièces. Enfin, l'identification dans cette parcelle d'un atelier de traitement de métal brut pendant la phase $250-300$ serait inédite à 
Besançon, l'activité de réduction ne semblant pas débuter dans la région avant la fin $\mathrm{du}_{\mathrm{IV}}^{\mathrm{e}}$ siècle.

Une autre activité, la fabrication de chaux, est représentée par deux fours postérieurs à 250, l'un construit à cheval sur le mur de façade de la parcelle 4 après son abandon et sur la rue, l'autre dans une petite pièce du balnéaire sud de la domus 11, également après son abandon. À cette période, seules les maisons des parcelles nord sont encore debout, parfois regroupées (reprise commune du mur de façade sur rue des parcelles 2 et 3 et des parcelles 10 et 9). Même si une réduction de l'habitat est avérée avec une occupation alors sans doute concentrée en bord de cardo, la présence des fours à chaux suggère que des activités de construction sont encore effectives (maisons ou mur de fortification).

\section{Signes de confort}

Ces maisons exhibent toutes des indices d'un certain confort, logique dans les domus résidentielles 11 (et 1 ?), mais également présent dans les domus plus petites tournées sur la rue. Les enduits peints décorent une partie des pièces de ces maisons. Des placages de marbre local ou importé sont présents entre 170 et 250 dans la grande halle de la parcelle 3 , entre 40 et 250 dans les parcelles 4 (marbre local) et 5 (marbres importés coûteux), dans les contextes de la seconde moitié du $\mathrm{II}^{\mathrm{e}}$ et du $\mathrm{III}^{\mathrm{e}}$ siècle des parcelles 6 (marbre local et Carrare) et 9 (marbres importés très coûteux), dans les niveaux tardifs de la parcelle 1 avec des marbres luxueux importés de Grèce, de Turquie et d'Afrique du Nord.

Des sols en béton de terrazzo sont construits dès 40 dans les parcelles 11 et 4 , à la période flavienne dans les parcelles 4, 5, 6 et 11, celui de la parcelle 6 étant orné d'un opus signinum. Ils ne sont plus de mode vers 150 (un seul, en parcelle 4), puis reviennent au début du III ${ }^{\mathrm{e}}$ siècle (parcelles 5 et 9) ou au milieu de ce siècle (parcelles 5 et 9). Au début du III $^{\mathrm{e}}$ siècle, la domus résidentielle 11 possède une grande pièce ornée d'une mosaïque (les quelques centimètres carrés conservés ne permettent cependant pas de la décrire) qui remplace une pièce sur hypocauste antérieure.

De nombreux foyers et cheminées participent au confort de ces maisons, tout comme un chauffage par hypocauste à canaux rayonnants attesté dans la parcelle 9 vers 170 et un hypocauste à pilettes et plateforme maçonnée pour supporter une baignoire (balnéaire) dans la parcelle 2 durant tout le III $^{\mathrm{e}}$ siècle [ill. 3]. La domus résidentielle 11 dispose d'un petit balnéaire aménagé à l'est de la parcelle dès sa construction [ill. 5], puis d'un balnéaire plus spacieux après l'agrandissement de la propriété vers le sud au début du $\mathrm{II}^{\mathrm{e}}$ siècle [ill. 2].

Létude de ce quartier de Vesontio évoluant sur la longue durée (quatre siècles) offre une image très dynamique des différentes maisons fouillées. La mixité entre les habitations et les activités de commerce et d'artisanat, toujours d'actualité dans le quartier Pasteur du XXI ${ }^{\mathrm{e}}$ siècle, est ici mise en évidence grâce au choix architectural et urbanistique des constructeurs de l'Antiquité (édification du quartier en une seule phase, homogénéité des constructions), puis au choix des aménagements intérieurs des acteurs impliqués au quotidien dans chacune des parcelles (multiplication des structures de combustion, espaces de stockage...). La comparaison avec les quartiers artisanaux et commerçants des agglomérations secondaires, en particulier celle de Bliesbruck (Petit, 2005), montre que le schéma dégagé dans ces contextes est également présent en capitale de cité.

\section{Références bibliographiques}

ADAM J.-P., 1989, La construction romaine, Paris, Picard, $368 \mathrm{p}$.

Berti Rossi S., May Castella C. (dir.), 2005, La fouille de Vidy «Chavannes $11 »(1989-1990)$, trois siècles d'histoire à Lousonna : archéologie, architecture et urbanisme, Lausanne, Bibliothèque historique vaudoise, «Cahiers d'archéologie romande » 102, $392 \mathrm{p}$.

BINET E. (dir.), 2010, Évolution d'une insula de Samarobriva au Haut-Empire, les fouilles du « Palai des Sports / Coliseum » à Amiens (Somme), Amiens, Revue archéologique de Picardie, $n^{\circ}$ spécial 27, 444 p.

Brissaud L. et al., 1999, Saint-Romain-en-Gal, Guide du site, Paris, Réunion des Musées Nationaux, $112 \mathrm{p}$

BRULET R., 1996, «La maison urbaine en Gaule Belgique et en Germanie inférieure », in La maison urbaine d'époque romaine en Gaule narbonnaise et dans les provinces voisines, Actes du colloque d'Avignon (11-13 nov. 1994), [Avignon], Association pour la promotion de la recherche archéologique en Vaucluse, «Documents d'Archéologie Vauclusienne » 6, p. 73-97

BRULET R. (dir.), 2008, Les romains en Wallonie, Bruxelles, Éditions Racine, 621 p.

Coquelet C., 2011, Les capitales de cité des provinces de Belgique et de Germanie, étude urbanistique, Louvain, Presses universitaires de Louvain, 366 p.
De Vos A. et M., 1982, Pompéi, Ercolano, Stabia, guide archeologiche Laterza, Roma, Laterza \& figli, $380 \mathrm{p}$

KerÉBEL H., 2001, Corseul (Côtes-d'Armor), un quartier de la ville antique, les fouilles de Monterfil II, Paris, Éd. de la Maison des sciences de l'homme, «Documents d'archéologie française » 88, 248 p.

Monteix N., 2010, Les lieux de métier, boutiques et ateliers d'Herculanum, Rome, École française de Rome, « Bibliothèque des Écoles françaises d'Athènes et de Rome » fasc. 344, Collection du Centre Jean Bérard 34, 478 p.

Munier C. (dir.), 2016, Besançon (Doubs), Zac Pasteur, Rapport d'opération, Ville de Besançon-Inrap-SRA Bourgogne-Franche-Comté, 11 vol., $4500 \mathrm{p}$.

Olivier A., 1988, « Rues et portiques de MalainMédiolanum : les problèmes d'une restitution ", Archeologia, $\mathrm{n}^{\circ}$ 237, p. 74-78.

Petit J.-P. (collab. Albrecht P.-A.), 2005, « L'artisanat alimentaire dans les petites villes gallo-romaines de Bliesbruck (France, département Moselle) et Schwarzenacker (Allemagne, Land de Sarre) au III siècle apr. J.-C. », in Polfer M. (dir.), Artisanat et économie romaine: Italie et provinces occidentales de l'Empire, actes du $3^{\mathrm{e}}$ colloque international d'Erpeldange (Luxembourg) sur l'artisanat romain (oct. 2004). Montagnac, éd. Monique Mergoil, p. 169-193
Petit J.-P., Santoro S., 2007, Vivre en Europe romaine, de Pompéi à Bliesbruck-Reinheim, Paris, Éd. Errance, $246 \mathrm{p}$.

Provost A., 2007, « La villa maritime de Mané-Véchen à Plouhinec (Morbihan) », in Aremorica, Études sur l'ouest de la Gaule romaine, $\mathrm{n}^{\circ}{ }_{1}$, Brest, CRBC, p. 85-100.

SAliou C., 1994, Les lois du bâtiment : voisinage et habitat urbain dans l'Empire romain, recherches sur les rapports entre le droit et la construction privée du siècle d'Auguste au siècle de Justinien, Beyrouth, Institut français d'archéologie du Proche-Orient, « Bibliothèque archéologique et historique » 116, $340 \mathrm{p}$.

Viscusi-Simonin V., 2016, Besançon (Doubs), Zac Pasteur, étude du bâti, Rapport d'opération, Inrap-Ville de Besançon-SRA BourgogneFranche-Comté, 2 vol., 504 p. 